\title{
SEMICLASSICAL RESONANCES VIA MEROMORPHY OF THE RESOLVENT AND THE $S$-MATRIX
}

\section{SOUMIA BELMOUHOUB ${ }^{1,2, *}$, BEKKAI MESSIRDI ${ }^{2}$ AND ABDERRAHMANE SENOUSSAOUI ${ }^{1,2}$}

\author{
${ }^{1}$ Department of Mathematics, Faculty of Exact and Applied Sciences, University of Oran1 Ahmed Ben \\ Bella, Algeria \\ ${ }^{2}$ Laboratory of Fundamental and Applicable Mathematics of Oran (LMFAO), University of Oran1 Ahmed \\ Ben Bella, Algeria
}

Corresponding author: belmsou@yahoo.fr

\begin{abstract}
The purpose of this paper is to describe the basic problems of resonances via meromorphic continuation of the resolvent and the scattering matrix. An example from mathematical physics is given by investigating the poles of the resolvent of semiclassical Schrödinger operators and Born-Oppenheimer Hamiltonians. Mathematical techniques, dilation-analyticity and Feshbach reduction are used here for the characterization of resonances of these Hamiltonians.
\end{abstract}

\section{INTRODUCTION}

The spectrum in the complex plane of Schrödinger operators $P(h)=-h^{2} \Delta+V$, is often the union of the line $\operatorname{Im} z=0$ and at most finitely-many points of the form $i \lambda_{j}(h)$ on the positive imaginary axis $\lambda_{j}(h)>0$. These points correspond to the negative eigenvalues of $P(h)$ so that $z(h)=-\lambda_{j}^{2}(h)$ belongs to the discrete spectrum of $P(h)$. The resolvent $\left(P(h)-\lambda^{2}\right)^{-1}$ is an operator-valued function defined for $\operatorname{Im} \lambda>0$ and $\lambda \neq \lambda_{j}(h)$. We would like to find the largest region in the complex $\lambda$-plane on which the

Received 2020-01-11; accepted 2020-01-28; published 2020-03-02.

2010 Mathematics Subject Classification. 35J10, 35P25, 47A56, 47A75.

Key words and phrases. Schrödinger operators; Born-Oppenheimer Hamiltonians; Meromorphic continuation; Resonances; Scattering matrix; Feshbach reduction; Dilation operator.

(C)2020 Authors retain the copyrights of their papers, and all open access articles are distributed under the terms of the Creative Commons Attribution License. 
resolvent can be defined. For several types of potential $V$, the spectrum of $-h^{2} \Delta+V$ is continuous and equals $[0,+\infty[$, and hence contains no (further) information about $V$. In this setting resonances replace the discrete data of eigenvalues. Precisely, the poles of the meromorphic continuation of the resolvent are called resonances or scattering poles. They constitute a natural remplacement of discrete spectral data for problems on non-compact domains. The multiplicity of a pole $\lambda_{0}$ is given in terms of multiplicity of the corresponding resonance $z_{0}=\lambda_{0}^{2}$, multiplicity of $\lambda_{0}=\operatorname{dim} \operatorname{Im} \pi_{\lambda_{0}^{2}}\left(L_{\text {comp }}^{2}\left(\mathbb{R}^{n}\right)\right)$ where:

$$
\begin{aligned}
& \pi_{\lambda_{0}^{2}}=\frac{1}{2 \pi i} \oint_{\gamma}(z-P(h))^{-1} d z: L_{c o m p}^{2}\left(\mathbb{R}^{n}\right) \longrightarrow L_{l o c}^{2}\left(\mathbb{R}^{n}\right) \\
& \gamma:\left[0,2 \pi\left[\ni t \mapsto z_{0}+\varepsilon e^{i t} .\right.\right.
\end{aligned}
$$

The resonances are shown to be the same as the poles of the meromorphically continued scattering matrix. Meromorphic extensions of resolvents have been studied in many frameworks and their finite rank poles or resonances, serve in a sense as discrete data similar in character to eigenvalues of a compact manifold. However, if the manifold has constant negative sectional curvature away from a compact, Guillopé and Zworski [1] showed the meromorphic continuation of the resolvent to $\mathbb{C}$ with finite rank poles. For $n$ odd they are defined as the poles of the meromorphic continuation of $\left(P(h)-\lambda^{2}\right)^{-1}: L_{\text {comp }}^{2}\left(\mathbb{R}^{n}\right) \longrightarrow L_{l o c}^{2}\left(\mathbb{R}^{n}\right)$ from $\{\operatorname{Im} \lambda>0\}$ to $\mathbb{C}$ or to the Riemann surface (logarithmic covering of $\mathbb{C}$ ) if $n$ is even. The main advantage of odd dimensions greater than one is the strong Huyghens principle for the wave equation. Effectively, one consequence of the strong Huyghens principle is the analytic continuation of $\left(-h^{2} \Delta-\lambda^{2}\right)^{-1}$ from $\{\operatorname{Im} \lambda>0\}$ to $\mathbb{C}$.

Under suitable assumptions on $V$, the operator $P(h)$ extends as a selfadjoint family of operators on $L^{2}\left(\mathbb{R}^{n}\right)$ with continuous spectrum $\left[0,+\infty\left[\right.\right.$, for example if $V$ is real-valued and $\lim _{|x| \rightarrow \infty} V(x)=0$. For $\lambda \in$ $\mathbb{C} \backslash\left[0,+\infty\left[\right.\right.$, the resolvent $R_{V}(\lambda)=(P(h)-\lambda)^{-1}$ is an holomorphic function from $\mathbb{C} \backslash\left[0,+\infty\left[\right.\right.$ to $\mathcal{B}\left(L^{2}\left(\mathbb{R}^{n}\right)\right)$ the algebra of bounded operators on $L^{2}\left(\mathbb{R}^{n}\right)$. As operator on $L^{2}\left(\mathbb{R}^{n}\right), R_{V}(\lambda)$ has no analytic extension across its spectrum. But, if we replace $L^{2}\left(\mathbb{R}^{n}\right)$ by a smaller dense subspace, like $C_{0}^{\infty}\left(\mathbb{R}^{n}\right)$, then $R_{V}(\lambda)$ might have some continuation across $[0,+\infty[$ to some Riemann surface above $\mathbb{C} \backslash[0,+\infty[$. If the continuation turns out to be meromorphic, we then obtain the resonance of $P(h)$ which are exactly the poles of this continuation. When $V=0$, i.e. $P(h)=-h^{2} \Delta$, is the free Hamiltonian, the resonances can be accessible using Fourier analysis. If $V \neq 0$, many effective approaches combine the known extension of the free resolvent to properties of $V$.

The mathematical study of resonances initiated for Schrödinger operators on $\mathbb{R}^{n}$. Later, it was extended to more geometric situations, such as the Laplacian on hyperbolic and asymptotically hyperbolic manifolds, symmetric or locally symmetric spaces, and Damek-Ricci spaces, see e.g. [1] and [2]. In a typical situation, 
one works on a complete Riemannian manifold $X$, for which the positive Laplacian $-\Delta$ is an essentially self-adjoint operator on the Hilbert space $L^{2}(X)$ of square integrable functions on $X$.

The basic problems of resonances are described here for Schrödinger operators and Born-Oppenheimer Hamiltonians with regular and singular potentials. We first show an holomorphic extension result via Fredholm operator theory in Hilbert spaces. In section 2, we review basic situations for meromorphic continuation of the resolvent. We study in section 3, the meromorphy of the scattering matrix, it follows that the poles of the meromorphic continuation of the $S$-matrix are exactly the poles of the continuation of the resolvent and conversely. Some interesting characterizations of the resonances of semiclassical Schrödinger operators and Hamiltonians in the Born-Oppenheimer approximation are obtained in section 4, by dilation-analyticity and Feshbach reduction.

We start with the following definition.

Definition 1.1. Let $\Omega \subseteq \mathbb{C}$ be open and connected and $H$ a complex Hilbert space. Suppose that $A(\lambda)$ is a $\mathcal{B}(H)$-valued analytic function on $\Omega$ except for isolated singularities. Then $A(\lambda)$ is said meromorphic in $\Omega$, if for each $\lambda_{0} \in \Omega$, there exist a neighbourhood $U_{\lambda_{0}}$ of $\lambda_{0}$, an integer $p>0$ and some $\left(A_{i}\right)_{1 \leq i \leq p} \subset \mathcal{B}(H)$ such that for all $\lambda \in U_{\lambda_{0}} \backslash\left\{\lambda_{0}\right\}$, we have the finite Laurent expansion:

$$
A(\lambda)=\sum_{i=1}^{p} \frac{A_{i}}{\left(\lambda-\lambda_{0}\right)^{i}}+B(\lambda)
$$

where $B(\lambda)$ is an holomorphic function on $U_{\lambda_{0}}$ with values in the algebra $\mathcal{B}(H)$ of bounded linear operators on $H$.

It is easy to see that $A(\lambda)$ is holomorphic in $U \backslash S$ where $S$ is a discrete set of $U$ whose elements are the poles of $A(\lambda) . p$ is the order of the pole, $A_{1}$ is the residue of $A(\lambda)$ at $\lambda_{0}$.

We essentially have the following result:

Theorem 1.1. Let $\Omega \subseteq \mathbb{C}$ be a connected open set and $(A(z))_{z \in \Omega}$ a holomorphic family of Fredholm operators on $H$. If $A\left(z_{0}\right)^{-1}$ exists at some $z_{0} \in \Omega$, then $z \mapsto A(z)^{-1}$ is a meromorphic family in $\Omega$ of operators with poles of finite rank.

Proof. For any $z \in \Omega$, let $n_{+}=\operatorname{dim} \operatorname{ker} A(z)$ and $n_{-}=\operatorname{dim} \frac{H}{\operatorname{ImA(z)}}$ and set $H_{+}=\mathbb{C}^{n_{+}}$and $H_{-}=\mathbb{C}^{n_{-}}$. Let $\left\{e_{1}, \ldots, e_{n_{+}}\right\}$be a basis of $H_{+}$. So define

$$
\begin{aligned}
R_{+} \quad: \quad H & \longrightarrow H_{+} \\
x & \mapsto R_{+} x=\left(\left\langle x, e_{1}\right\rangle, \ldots,\left\langle x, e_{n_{+}}\right\rangle\right) .
\end{aligned}
$$


Next choose $y_{1}, \ldots, y_{n_{-}}$whose images in $\frac{H}{\operatorname{Im} A(z)}$ form a basis of $\frac{H}{\operatorname{ImA(z)}}$ and define

$$
\begin{aligned}
R_{-} \quad: \quad & H_{-} \longrightarrow H \\
& x \mapsto R_{-}\left(a_{1}, \ldots, a_{n_{-}}\right)=\sum_{k=1}^{n_{-}} a_{k} y_{k} .
\end{aligned}
$$

We produce a Grushin problem for $A(z)$ as decribed by Belmouhoub and Messirdi in [3]:

$$
\mathcal{A}(z)=\left(\begin{array}{cc}
A(z) & R_{-} \\
R_{+} & 0
\end{array}\right) .
$$

$\mathcal{A}(z)$ is well-posed Grushin problem for $z$ in some sufficiently small neighborhood $V(z)$ of $z$ with inverse

$$
\mathcal{E}(z)=\left(\begin{array}{cc}
E(z) & E_{+}(z) \\
E_{-}(z) & E_{-+}(z)
\end{array}\right): H \oplus H_{+} \longrightarrow H \oplus H_{-}
$$

such that $A(z)$ is invertible if and only if $E_{-+}(z)$ is invertible and

$$
\left\{\begin{array}{l}
A^{-1}(z)=E(z)-E_{+}(z) E_{-+}^{-1}(z) E_{-}(z) \\
E_{-+}^{-1}(z)=-R_{+} A(z)^{-1} R_{-}
\end{array}\right.
$$

In particular, this is true for $z=z_{0}$ and for $z \in V(z)$. We know that the index of $A(z)$ is constant in $V(z)$. Since the index vanishes at $z_{0}$, then at any $z$ we have $n_{+}=n_{-}=n$ and $E_{-+}(z)$ is an $n \times n$ matrix with holomorphic coefficients. So for any $z \in \Omega$ the function $f_{z}(\lambda)=\operatorname{det} E_{-+}(\lambda)$ is holomorphic in a neighborhood of $z$ such that $A(\lambda)$ is invertible if and only if $f_{z}(\lambda) \neq 0$. As $\Omega$ is connected and since $A\left(z_{0}\right)$ is invertible for at least one $z_{0} \in \Omega$, none of the functions $f_{z}$ can be identically zero. Since $\operatorname{det} E_{-+}(z)$ is not identically zero, $E_{-+}(z)$ is a meromorphic family of matrices. From the Schur complement (1.1), then $z \mapsto A(z)^{-1}$ is a meromorphic family of operators with poles of finite rank.

\section{Meromorphic CONTINUAtion of the RESOlvent}

2.1. Helmholtz operator. Consider the resolvent of Laplacian $\Delta=\sum_{k=1}^{n} \frac{\partial^{2}}{\partial x_{k}^{2}}$ defined by:

$$
R_{0}(\lambda)=\left(-h^{2} \Delta-\lambda^{2}\right)^{-1}: L^{2}\left(\mathbb{R}^{n}\right) \longrightarrow L^{2}\left(\mathbb{R}^{n}\right) \text { for } \operatorname{Im} \lambda>0, h>0 .
$$

Since the classical theorems on the usual Fourier transform extend to the semiclassical case, it suffices to consider $h=1$. Then the existence of $R_{0}(\lambda)$ follows from using the Fourier transform which provides an explicit diagonalization of $-\Delta$ :

$$
R_{0}(\lambda) u(x)=\frac{1}{(2 \pi)^{n}} \int_{\mathbb{R}^{n}} \frac{e^{i x \xi}}{|\xi|^{2}-\lambda^{2}} \widehat{u}(\xi) d \xi, \operatorname{Im} \lambda>0 .
$$

(2.1) is of course valid in all dimension but the resolvent operator $R_{0}(\lambda)$ has much nice properties when $n$ is odd. We establish the following important result concerning the meromorphic continuation of the resolvent in odd dimensions. 
Theorem 2.1. ( [4]) Suppose that $n \geq 3$ is odd, then the resolvent operator $R_{0}(\lambda)$ defined on $L^{2}\left(\mathbb{R}^{n}\right)$ for $\operatorname{Im} \lambda>0$, extends analytically to an entire family of operators from $L_{\text {comp }}^{2}\left(\mathbb{R}^{n}\right)$ to $L_{\text {loc }}^{2}\left(\mathbb{R}^{n}\right)$.

For any $\chi \in C_{0}^{\infty}\left(\mathbb{R}^{n}\right)$ :

$$
\left\|\chi R_{0}(\lambda) \chi\right\|_{L^{2}\left(\mathbb{R}^{n}\right) \rightarrow H^{k}\left(\mathbb{R}^{n}\right)}=\mathcal{O}\left((1+|\lambda|)^{k-1} e^{\max (-\operatorname{Im} \lambda, 0) R}\right),
$$

$k=0,1,2$ and $\operatorname{supp}(\chi) \subset B(0, R)$ (the open ball of radius $R$ centered at 0 ).

Proof. By the functional calculus, we have (see e.g. [4]):

$$
R_{0}(\lambda)=\int_{0}^{+\infty} e^{i \lambda t} U(t) d t, \operatorname{Im} \lambda>0
$$

where $U(t)=\frac{\sin (t \sqrt{-\Delta})}{\sqrt{-\Delta}}$. Now $\frac{\partial^{2} U}{\partial t^{2}}=\Delta U(t)$ so $U(t)$ is a operator solution of the wave equation. More precisely,

$$
\begin{aligned}
u(x, t) & =U(t) \phi_{1}(x)+U^{\prime}(t) \phi_{0}(x) \\
& =\frac{\sin (t \sqrt{-\Delta})}{\sqrt{-\Delta}} \phi_{1}(x)+\cos (t \sqrt{-\Delta}) \phi_{0}(x)
\end{aligned}
$$

is the solution of the wave equation with the initial conditions $u(x, 0)=\phi_{0}(x)$ and $\frac{\partial u}{\partial t}(x, 0)=\phi_{1}(x)$. In odd dimensions, the strong Huyghens principle (see [5]) implies that:

$$
\operatorname{supp} f \subset B(0, R) \Longrightarrow(U(t) f)(x)=0,|x|<t-R .
$$

So, if $\chi \in C_{0}^{\infty}\left(\mathbb{R}^{n}\right)$ with $\operatorname{supp}(\chi) \subset B(0, R)$, then:

$$
\chi R_{0}(\lambda) \chi=\int_{0}^{2 R} e^{i \lambda t} \chi U(t) \chi d t, \operatorname{Im} \lambda>0 .
$$

The right hand side is now defined and, as an operator $L^{2}\left(\mathbb{R}^{n}\right) \longrightarrow L^{2}\left(\mathbb{R}^{n}\right)$, holomorphic for $\lambda \in \mathbb{C}$. In fact,

$$
\lambda \chi R_{0}(\lambda) \chi=\int_{0}^{2 R} D_{t}\left(e^{i \lambda t}\right) \chi U(t) \chi d t,
$$

where $D_{t}=\frac{1}{i} \frac{\partial}{\partial t}$. Since $D_{t} U(t)=\frac{1}{i} \cos (t \sqrt{-\Delta})$, integration by parts shows that the right hand side is bounded with a bound depending on $R$ and $\alpha=\max (-\operatorname{Im} \lambda, 0)$. So,

$$
\left\|\chi R_{0}(\lambda) \chi\right\|_{L^{2} \rightarrow L^{2}} \leq \frac{e^{\alpha R}}{|\lambda|} .
$$

We can consider $U(t)$ as a map from $L^{2}\left(\mathbb{R}^{n}\right)$ to the Sobolev space $H^{1}\left(\mathbb{R}^{n}\right)$. Indeed, since $\sup _{\lambda \in \mathbb{R}}\left|\frac{\sin t \lambda}{\lambda}\right|=|t|$, we have:

$$
\|U(t)\|_{L^{2} \rightarrow H^{1}}=\|U(t)\|_{L^{2} \rightarrow L^{2}}+\|\sqrt{-\Delta} U(t)\|_{L^{2} \rightarrow L^{2}}=\mathcal{O}(|t|)+\mathcal{O}(1)
$$

and integrating shows that:

$$
\left\|\chi R_{0}(\lambda) \chi\right\|_{L^{2} \rightarrow H^{1}}=\mathcal{O}\left(e^{\alpha R}\right) .
$$


We also get a bound for $\chi R_{0}(\lambda) \chi$ as a map from $L^{2}\left(\mathbb{R}^{n}\right)$ to $H^{2}\left(\mathbb{R}^{n}\right)$. Recall that the norm on $H^{2}\left(\mathbb{R}^{n}\right)$ can be taken as $\|u\|_{L^{2}}+\|\Delta u\|_{L^{2}}$. So, we have:

$$
\begin{aligned}
\left\|\chi R_{0}(\lambda) \chi\right\|_{L^{2} \rightarrow H^{2}} \leq & \left\|\Delta\left(\chi R_{0}(\lambda) \chi\right)\right\|_{L^{2} \rightarrow L^{2}}+\left\|\chi R_{0}(\lambda) \chi\right\|_{L^{2} \rightarrow L^{2}} \\
\leq & \left\|\chi \Delta\left(R_{0}(\lambda) \chi\right)\right\|_{L^{2} \rightarrow L^{2}}+\left\|[\Delta, \chi]\left(\chi_{1} R_{0}(\lambda) \chi_{1}\right) \chi\right\|_{L^{2} \rightarrow L^{2}} \\
& +\left\|\chi R_{0}(\lambda) \chi\right\|_{L^{2} \rightarrow L^{2}}
\end{aligned}
$$

where $\chi_{1} \in C_{0}^{\infty}\left(\mathbb{R}^{n}\right)$ such that $\chi_{1}=1$ near $\operatorname{supp}(\chi)$ and with supp $\chi_{1}$ in a ball of radius $R_{1}>R$. Since $\left(-\Delta-\lambda^{2}\right) R_{0}(\lambda)=I_{L^{2}\left(\mathbb{R}^{n}\right)}$ (as operators on functions of compact support), we have:

$$
\left\|\chi \Delta\left(R_{0}(\lambda) \chi\right)\right\|_{L^{2} \rightarrow L^{2}}=\mathcal{O}\left(\lambda^{2}\right)
$$

Since $[\Delta, \chi]$ is a first order operator, we obtain:

$$
\left\|[\Delta, \chi]\left(\chi_{1} R_{0}(\lambda) \chi_{1}\right) \chi\right\|_{L^{2} \rightarrow L^{2}} \leq C\left\|\chi_{1} R_{0}(\lambda) \chi_{1}\right\|_{L^{2} \rightarrow H^{1}}, C>0
$$

So

$$
\begin{aligned}
\left\|\chi R_{0}(\lambda) \chi\right\|_{L^{2} \rightarrow H^{2}} \leq & |\lambda|^{2}\left\|\chi R_{0}(\lambda) \chi\right\|_{L^{2} \rightarrow L^{2}}+C\left\|\chi_{1} R_{0}(\lambda) \chi_{1}\right\|_{L^{2} \rightarrow H^{1}} \\
& +\left\|\chi R_{0}(\lambda) \chi\right\|_{L^{2} \rightarrow L^{2}} .
\end{aligned}
$$

$\chi R_{0}(\lambda) \chi$ is a bounded operator from $L^{2}\left(\mathbb{R}^{n}\right)$ to $L^{2}\left(\mathbb{R}^{n}\right)$ and its image consists of functions supported in $B(0, R)$. By Rellich's lemma, the embedding of this space in $L^{2}\left(\mathbb{R}^{n}\right)$ is compact. Hence:

$$
\left\|\chi R_{0}(\lambda) \chi\right\|_{L^{2} \rightarrow H^{2}}=\mathcal{O}\left(|\lambda| e^{\alpha R_{1}}\right)
$$

Remark 2.1. Suppose $n$ is odd and $R_{0}(\lambda): L^{2}\left(\mathbb{R}^{n}\right) \longrightarrow L^{2}\left(\mathbb{R}^{n}\right)$ for $\operatorname{Im} \lambda>0$. Then the analytic continuation of the Schwartz kernel $R_{0}(\lambda, x, y)$ is given by Stone's formula [6]:

$$
R_{0}(\lambda, x, y)-R_{0}(-\lambda, x, y)=\frac{i}{2} \frac{\lambda^{n-2}}{(2 \pi)^{n-1}} \int_{S^{n}} e^{i \lambda \omega(x-y)} d \omega, \lambda \in \mathbb{C}
$$

where $d \omega$ denotes the standard measure on the unit sphere $S^{n}$ of $\mathbb{R}^{n}$.

2.2. Schrödinger operators. Let's study now the resolvent of the Schrödinger operator $P=-\Delta+V$ on $L^{2}\left(\mathbb{R}^{n}\right)$ with domain $H^{2}\left(\mathbb{R}^{n}\right)$ where $V \in L^{\infty}\left(\mathbb{R}^{n}, \mathbb{C}\right), n \geq 3$, odd. The resolvent operator $R_{V}(\lambda)=\left(P-\lambda^{2}\right)^{-1}$ exists at points $\lambda \in \mathbb{C}$ such that $\operatorname{Im} \lambda \gg 0$.

Theorem 2.2. (Meromorphic continuation of the resolvent) Suppose that $V \in L_{\text {comp }}^{\infty}\left(\mathbb{R}^{n}, \mathbb{C}\right.$ ) (ie $V$ is a.e. bounded potential of compact support), $n \geq 3$ is odd. Then 
1) $R_{V}(\lambda): L^{2}\left(\mathbb{R}^{n}\right) \longrightarrow L^{2}\left(\mathbb{R}^{n}\right)$ for $\operatorname{Im} \lambda>0$, is a meromorphic family of operators with finitely many poles.

2) $R_{V}(\lambda)$ extends to a meromorphic family of operators $R_{V}(\lambda): L_{\text {comp }}^{2}\left(\mathbb{R}^{n}\right) \longrightarrow L_{\text {loc }}^{2}\left(\mathbb{R}^{n}\right)$ for $\lambda \in \mathbb{C}$.

3) If $\chi \in C_{0}^{\infty}\left(\mathbb{R}^{n}\right)$ then $\left\|\chi R_{V}(\lambda) \chi\right\|_{L^{2} \rightarrow L^{2}} \leq \frac{C}{\lambda}, \lambda>0$.

Proof. We write:

$$
R_{V}(\lambda)-R_{0}(\lambda)=\left(-\Delta+V-\lambda^{2}\right)-\left(-\Delta-\lambda^{2}\right)=-R_{V}(\lambda) V R_{0}(\lambda)
$$

where $\chi \in C_{0}^{\infty}\left(\mathbb{R}^{n}\right)$ such that $\chi V=V$. Multiply the above equation on the right by $\chi$ to get

$$
R_{V}(\lambda) \chi-R_{0}(\lambda) \chi=-R_{V}(\lambda) \chi V R_{0}(\lambda) \chi
$$

so

$$
R_{V}(\lambda) \chi\left(I+V R_{0}(\lambda) \chi\right)=R_{0}(\lambda) \chi
$$

But, $\left\|R_{0}(\lambda)\right\|_{L^{2} \rightarrow L^{2}} \leq \frac{1}{|\operatorname{Im} \lambda|^{2}}$, so for large values of $\operatorname{Im} \lambda,\left(I+V R_{0}(\lambda) \chi\right)$ is invertible and

$$
R_{V}(\lambda)-R_{0}(\lambda)=-R_{0}(\lambda)\left(I+V R_{0}(\lambda) \chi\right)^{-1} V R_{0}(\lambda)
$$

Now $V R_{0}(\lambda) \chi=V \chi R_{0}(\lambda) \chi$ is compact being the product of a bounded operator with a compact operator. Then, $V R_{0}(\lambda) \chi$ is analytic family of compact operators and we can apply the analytic Fredholm theory (Theorem 1.2) to conclude that $R_{V}(\lambda)$ extends as a meromorphic operator valued family of operators $L_{\text {comp }}^{2}\left(\mathbb{R}^{n}\right) \longrightarrow L_{\text {loc }}^{2}\left(\mathbb{R}^{n}\right)$ for $\lambda \in \mathbb{C}$.

On the other hand, we have shown that $R_{V}(\lambda) \chi=R_{0}(\lambda)\left(I+V R_{0}(\lambda) \chi\right)^{-1}$ is a meromorphic family of operators. For $\lambda \gg 1$, we have $\left\|\left(I+V R_{0}(\lambda) \chi\right)^{-1}\right\|_{L^{2} \rightarrow L^{2}} \leq 2$ and hence the estimate on $R_{0}(\lambda)$ implies part (3) of the theorem.

Remark 2.2. In the even-dimensional case similar results are valid, except that the resolvent operator only extends to be entire on the logarithmic covering of the complex plane. Precisely, for $n$ even, $R_{V}(\lambda)$ extends to be entire as a function of $\log \lambda$, i.e. entire on the logarithmic covering $\Lambda$ of $\mathbb{C}$.

\section{Meromorphy of THE SCATTERING MATRIX}

We have just studied above the meromorphic continuation of the resolvent to $\mathbb{C}$ for odd dimensions and to $\Lambda$ for even dimensions. From this it can be deduced that the scattering matrix has a similar continuation. Indeed, the meromorphic continuation of the cut-off resolvent $\chi R_{V}(\lambda) \chi$ permits us to mermorphically continue the scattering matrix $S(\lambda)$ as a bounded operator on $L_{\text {loc }}^{2}\left(S^{n}\right)$ on $\mathbb{C}$ or on $\Lambda$ depending on the parity of $n$. In this section, we will define and describe the scattering matrix of $P(h)=-h^{2} \Delta+V$ for $V \in L_{\text {comp }}^{\infty}\left(\mathbb{R}^{n}, \mathbb{R}\right), n \geq 3$, where $L_{\text {comp }}^{\infty}\left(\mathbb{R}^{n}, \mathbb{R}\right)$ is the space of real essentially bounded functions of compact 
support. $P(h)$ is self-adjoint with domain $H^{2}\left(\mathbb{R}^{n}\right)$ and generates a one-parameter strongly continuous unitary group $\mathbb{R} \ni t \mapsto U_{V}(t)=e^{-i t P(h)}$. The unitary group $U_{V}(t)$ provides solutions $\psi(t)=U_{V}(t) \psi_{0}$, to the initial value problem:

$$
\left\{\begin{array}{l}
i \frac{\partial \psi}{\partial t}=P(h) \psi \\
\psi(0)=\psi_{0} \in H^{2}\left(\mathbb{R}^{n}\right)
\end{array}\right.
$$

$U_{0}(t)$ is the one-parameter strongly continuous unitary group associated to $-h^{2} \Delta$.

Proposition 3.1. 1) For any $f \in L^{2}\left(\mathbb{R}^{n}\right), n \geq 3$, the limits $\lim _{t \rightarrow \pm \infty} U_{V}(t)^{*} U_{0}(t) f$ exist and define bounded transformations $\Omega_{ \pm}(P(h),-\Delta)$ or $\Omega_{ \pm}$called wave operators:

$$
\begin{gathered}
\Omega_{ \pm} f=\lim _{t \rightarrow \pm \infty} U_{V}(t)^{*} U_{0}(t) f \\
\left\|\Omega_{ \pm}\right\|_{L^{2} \rightarrow L^{2}}=1
\end{gathered}
$$

2) For any $f, g \in L^{2}\left(\mathbb{R}^{n}\right)$,

$$
\begin{gathered}
\left\langle\Omega_{ \pm} f, \Omega_{ \pm} g\right\rangle_{L^{2}}=\langle f, g\rangle_{L^{2}} \\
\left(\Omega_{ \pm}^{*}\right)^{*} \Omega_{ \pm}^{*}=\Omega_{ \pm} \Omega_{ \pm}^{*}
\end{gathered}
$$

3) The operator $F_{ \pm}=\Omega_{ \pm} \Omega_{ \pm}^{*}$ satisfies:

$$
\begin{gathered}
F_{ \pm}^{2}=F_{ \pm}, F_{ \pm}^{*}=F_{ \pm}, \quad F_{ \pm} \Omega_{ \pm}^{*}=\Omega_{ \pm}^{*},\left\|\Omega_{ \pm}^{*} f\right\|=\left\|F_{ \pm} f\right\|, \operatorname{Im} \Omega_{ \pm}=\operatorname{Im} F_{ \pm} . \\
\Omega_{ \pm} U_{0}(t)=U_{V}(t) \Omega_{ \pm} \text {and } U_{0}(t) \Omega_{ \pm}^{*}=\Omega_{ \pm}^{*} U_{V}(t) .
\end{gathered}
$$

4) The pair $(-\Delta, P(h))$ is asymptotically complete in the sense that the wave operators $\Omega_{ \pm}(-\Delta, P(h))$ exist.

Proof. The existence of wave operators comes from an explicit estimate for the free propagation given by $U_{0}(t)$ (see e.g. [7]). The relations (2), (3) and (4) follow from the existence of $\Omega_{ \pm}$and the simple properties of the unitary evolution groups.

The existence of the wave operators $\Omega_{ \pm}$gives the limits $\lim _{t \rightarrow \pm \infty} U_{V}(t)^{*} U_{0}(t) f=f_{ \pm}$. The scattering operator $S$ maps $f_{-}$to $f_{+}$. It is a bounded operator on $L^{2}\left(\mathbb{R}^{n}\right)$ since

$$
\begin{aligned}
& S f_{-}=f_{+}=\Omega_{+}^{*} f=\Omega_{+}^{*} \Omega_{-} f_{-}, \\
& S=\Omega_{+}^{*} \Omega_{-}: L^{2}\left(\mathbb{R}^{n}\right) \longrightarrow L^{2}\left(\mathbb{R}^{n}\right) .
\end{aligned}
$$

Furthermore, the $S$-operator commutes with the free time evolution $U_{0}(t)$ :

$$
\begin{aligned}
S U_{0}(t) & =\Omega_{+}^{*} \Omega_{-} U_{0}(t)=\Omega_{+}^{*} U_{V}(t) \Omega_{-}=\left(U_{V}(-t) \Omega_{+}\right)^{*} \Omega_{-} \\
& =\left(\Omega_{+} U_{0}(-t)\right)^{*} \Omega_{-}=U_{0}(t) S .
\end{aligned}
$$


Remark 3.1. It's simple to show that if $\operatorname{Im} \Omega_{-}(P(h),-\Delta)=\operatorname{Im} \Omega_{+}(P(h),-\Delta)$, then, the $S$-operator is a unitary operator on $L^{2}\left(\mathbb{R}^{n}\right)$. Hence, the $S$-operator is invertible and $S^{-1}=S^{*}$.

This allows for a reduction of the $S$-operator to a family of operators $S(\lambda)$ defined on $L^{2}\left(S^{n}\right)$ called the $S$-matrix. Effectively, for $\lambda \in \mathbb{R}$ we can define the scattering operator $S(\lambda): L^{2}\left(S^{n}\right) \longrightarrow L^{2}\left(S^{n}\right)$ by (see [2]):

$$
S(\lambda)=I_{L^{2}\left(S^{n}\right)}+A(\lambda)
$$

with a trace class operator $A(\lambda)$, and it continues meromorphically to the entire complex plane. Its poles coincide with the poles of the resolvent with multiplicities, $m_{S}(\lambda)$, related to the multiplicities of the poles of the resolvent, $m_{R}(\lambda)$, by the formula $m_{S}(\lambda)=m_{R}(\lambda)-m_{R}(-\lambda)$. Which gives the following fundamental result:

Theorem 3.1. The scattering matrix $S(\lambda)$ admits a meromorphic continuation to $\mathbb{C}$ if $n$ is odd, or to the Riemann surface $\Lambda$, if $n$ is even, with poles precisely at the resonances of $P(h)$. The multiplicity of the poles are the same as the multiplicity of the poles for the resolvent of $P(h)$ and the residues at these poles have the same finite rank.

Remark 3.2. For the semiclassical Schrödinger operator $P(h)$, the resonances may be defined as the poles of the meromorphic continuation of the resolvent $R_{V}(\lambda)$ or in terms of the meromorphic continuation of the S-matrix $S(\lambda)$. From [8], it follows that the poles of the meromorphic continuation of the $S$-matrix are exactly the poles of the continuation of the resolvent and conversely. However, the scattering poles differ from the resolvent poles for example for hyperbolic spaces.

\section{Resonances of Semiclassical Schrödinger Operators and Born-Oppenheimer}

\section{HAMILTONIANS}

There are various models for which one can prove the existence of resonances for example that of Stark hydrogen Schrödinger operator, and also those of semiclassical approximation and especially the resonances in the Born-Oppenheimer approximation.

4.1. Resonances of semiclassical Schrödinger operators. The theory developed by Hunziker [9], identify the resonances with the eigenvalues of the deformed hamiltonian $P_{\theta}(h)$, in the lower complex half-plane, of the Schrödinger operator $P(h)=-h^{2} \Delta+V(x)$ defined on $L^{2}\left(\mathbb{R}^{n}\right)$ with domain $D(P(h))=H^{2}\left(\mathbb{R}^{n}\right) \cap D(V)$. The resonances do not depend on $\theta$ and they are associated with the poles of the meromorphic extension from the upper complex half-plane of the resolvent of $P_{\theta}(h)$. In order to prove the existence of such continuation we operate an explicit construction assuming appropriate conditions on the potential.

Let the potential $V(x)$ be smooth real function, extends analytically in $|\operatorname{Im} \theta|<\delta_{0}, \delta_{0}>0$, and such that $V(-\Delta+1)^{-1}$ is compact. We introduce the resonances of $P(h)=-h^{2} \Delta+V(x)$ on $L^{2}\left(\mathbb{R}^{n}\right)$ by using the 
analytic dilation operator:

$$
\left(U_{\theta} \varphi\right)(x)=e^{n \theta / 2} \varphi\left(e^{\theta} x\right), \varphi \in C_{0}^{\infty}\left(\mathbb{R}^{n}\right) .
$$

$U_{\theta}$ has a unitary extension on $L^{2}\left(\mathbb{R}^{n}\right)$. It follows that $V\left(e^{\theta} x\right)(-\Delta+1)^{-1}$ is a compact operator-valued analytic function of $\theta$ in the strip $|\operatorname{Im} \theta|<\delta_{0}$. Then,

$$
P_{\theta}(h)=U_{\theta}^{*} P(h) U_{\theta}=-h^{2} e^{-2 \theta} \Delta+V\left(e^{\theta} x\right)
$$

is an analytic family of non selfadjoint operators where $\theta$ runs in the strip $|\operatorname{Im} \theta|<\delta_{0}$, since for $z \in \mathbb{C}^{+}$ and $\varphi, \psi \in L^{2}\left(\mathbb{R}^{n}\right)$,

$$
\langle R(z) \varphi, \psi\rangle=\left\langle U_{\theta} R(z) \varphi, U_{\theta} \psi\right\rangle=\left\langle\left[U_{\theta} R(z) U_{\theta}^{*}\right] U_{\theta} \varphi, U_{\theta} \psi\right\rangle,
$$

where $U_{\theta} R(z) U_{\theta}^{*}=R_{\theta}(z)=\left(P_{\theta}(h)-z\right)^{-1}$.

Let $\sigma_{\text {ess }}=\sigma \backslash \sigma_{\text {disc }}$ be the essential spectrum where the discrete spectrum $\sigma_{\text {disc }}$ is the set of isolated points of the spectrum such that the corresponding Riesz projectors are finite dimensional. Then, by Weyl Theorem:

$$
\sigma_{\text {ess }}\left(P_{\theta}(h)\right)=e^{-2 \theta} \sigma_{\text {ess }}\left(-h^{2} \Delta+e^{2 \theta} V\left(e^{\theta} x\right)\right)=e^{-2 \theta} \sigma_{\text {ess }}\left(-h^{2} \Delta\right)=e^{-2 \theta} \mathbb{R}_{+} .
$$

The definition of resonances is adapted here as follows:

Definition 4.1. A complex number $\rho$ is a resonance of $P(h)$ if $\operatorname{Re} \rho>\inf \sigma_{\text {ess }}(P(h))$ and if there exists $\theta$ small enough, $\operatorname{Im} \theta>0$, such that $\rho \in \sigma_{\text {disc }}\left(P_{\theta}(h)\right) . \Sigma(h)$ denotes the set of resonances of $P(h)$.

It is well known, see for example the works of Messirdi [10], [11], that the resolvent operator $R(z)=$ $(P(h)-z)^{-1}, z \in \mathbb{C} \backslash \mathbb{R}$, admits an analytic extension in $\mathbb{C}^{+}=\{z \in \mathbb{C}: \operatorname{Im} z>0\}$ and under the assumption that $V\left(e^{\theta} x\right)$ is analytic, we can extend $R(z)$ to a meromorphic function in a larger domain, the set of poles of this extension is precisely $\Sigma(h)$. Effectively,

$$
\begin{aligned}
P_{\theta}(h)-z & =-h^{2} e^{-2 \theta} \Delta+V\left(e^{\theta} x\right)-z \\
& =\left[I+V\left(e^{\theta} x\right)\left(-h^{2} e^{-2 \theta} \Delta-z\right)^{-1}\right]\left(-h^{2} e^{-2 \theta} \Delta-z\right) .
\end{aligned}
$$

$\left[I+V\left(e^{\theta} x\right)\left(-h^{2} e^{-2 \theta} \Delta-z\right)^{-1}\right]$ is invertible for $|\operatorname{Im} z| \rightarrow \infty$ and $\left(-h^{2} e^{-2 \theta} \Delta-z\right)^{-1} \in \mathcal{B}\left(L^{2}\left(\mathbb{R}^{n}\right)\right)$ if $z \in \mathbb{C} \backslash e^{-2 \theta} \mathbb{R}_{+}$. Furthermore, it is easily shown that

$$
\frac{\partial}{\partial \bar{\theta}}\left[\left\langle R_{\theta}(z) U_{\theta} \varphi, U_{\bar{\theta}} \psi\right\rangle\right]=0
$$

(4.2) implies that $\left\langle R_{\theta}(z) U_{\theta} \varphi, U_{\bar{\theta}} \psi\right\rangle$ is an holomorphic function, for all $\varphi, \psi \in H^{2}\left(\mathbb{R}^{n}\right)$ and $z \in \Omega_{\delta}$ an non-empty open subset of $\mathbb{C}^{+}$such that $\Omega_{\delta} \cap \sigma\left(P_{\theta}(h)\right)=\emptyset,|\theta|<\delta_{0}$. However, (4.1) is true for $\theta \in \mathbb{R}$ and $z \in \mathbb{C}^{+}$, the two sides of this equality are holomorphic with respect to $\theta \in\left\{|\theta|<\delta_{0}\right\}$. The two holomorphic functions $\langle R(z) \varphi, \psi\rangle$ and $\left\langle U_{\theta} R(z) \varphi, U_{\theta} \psi\right\rangle$ coincide on a subset of $\mathbb{R}$, so equality is still true in $\left\{|\theta|<\delta_{0}\right\}$. Now consider (4.1) with respect to the variable $z$, the function $z \longmapsto R_{\theta}(z)$ is meromorphic on $\mathbb{C} \backslash \sigma_{\text {ess }}\left(P_{\theta}(h)\right)=$ 
$\mathbb{C} \backslash e^{-2 \theta} \mathbb{R}_{+}$, and the poles of $R_{\theta}(z)$ are exactly the elements of $\sigma_{d i s c}\left(P_{\theta}(h)\right)$. More precisely, we have for all $\theta$ complex such that $|\theta|<\delta_{0}$,

$$
\begin{aligned}
& \sigma_{\text {disc }}\left(P_{\theta}(h)\right)= \\
& \bigcup_{\varphi, \psi}\{\text { poles of } z \longmapsto\langle R(z) \varphi, \psi\rangle\} \cap\left\{z \in \mathbb{C}:-2 \operatorname{Im} \theta<\arg z<\frac{\pi}{2}\right\}
\end{aligned}
$$

and

$$
\Sigma(h)=\bigcup_{\operatorname{Im} \theta>0,|\theta|<\delta_{0}} \sigma_{d i s c}\left(P_{\theta}(h)\right) .
$$

4.2. Resonances of Born-Oppenheimer Hamiltonians. The quantum Hamiltonian in the BornOppenheimer approximation is written as:

$$
P(h)=-h^{2} \Delta_{x}+Q(x) ; Q(x)=-\Delta_{y}+V(x, y)
$$

on $L^{2}\left(\mathbb{R}_{x}^{n} \times \mathbb{R}_{y}^{p}\right)$ when $h$ tends to $0^{+} . \Delta_{x}$ (resp. $\Delta_{y}$ ) is the Laplace operator with respect to $x$ (resp. $y$ ), $x \in \mathbb{R}^{n}$ and $y \in \mathbb{R}^{p}, n=3 m, p=3 q, m, q \geq 1 . Q(x)$ is the electronic Hamiltonian defined on $L^{2}\left(\mathbb{R}_{y}^{p}\right)$. The purpose of this section is to show that using a general dilation operator:

$$
U_{\theta} \varphi(x, y)=e^{n \theta / 2} \varphi\left(e^{\theta} x, y\right), \varphi \in C_{0}^{\infty}\left(\mathbb{R}^{n} \times \mathbb{R}^{p}\right)
$$

and the Feshbach reduction scheme, the study of resonances of $P(h)$ is reduced to the discrete spectrum of a matrix of operators $F^{\theta}(z)$ defined on $L^{2}\left(\mathbb{R}_{x}^{n}\right) \oplus L^{2}\left(\mathbb{R}_{x}^{n}\right)$ (the so-called effective Hamiltonian) such that:

$$
z \text { is a resonance of } P(h) \Longleftrightarrow \exists \theta \in \mathbb{C}, \operatorname{Im} \theta>0, z \in \sigma_{\text {disc }}\left(F^{\theta}(z)\right) \text {. }
$$

The dilated Hamiltonian is

$$
P_{\theta}(h)=U_{\theta}^{*} P(h) U_{\theta}=-h^{2} e^{-2 \theta} \Delta_{x}+Q\left(e^{\theta} x\right)
$$

Assume that:

(H1) $V \in L^{\infty}\left(\mathbb{R}_{x}^{n} \times \mathbb{R}_{y}^{p}, \mathbb{R}\right)$ and can be analytically extended on the complex strip:

$$
D_{\delta_{0}}=\left\{x \in \mathbb{C}^{n}:|\operatorname{Im} x|<\delta_{0}\left(1+|\operatorname{Re} x|^{2}\right)^{1 / 2}\right\} .
$$

Thus, $P(h)$ and $Q(x)$ are selfadjoint on their respective natural domains $H^{2}\left(\mathbb{R}_{x}^{n} \times \mathbb{R}_{y}^{p}\right)$ and $H^{2}\left(\mathbb{R}_{y}^{p}\right)$. In particular, the domain of $Q(x)$ is independent of $x$. We suppose furthermore:

(H2) For every $x \in \mathbb{R}^{n}$, the spectrum of $Q(x)$ contains at least two eigenvalues $\lambda_{1}(x)$ and $\lambda_{2}(x)$ where $\lambda_{2}(x)$ is simple and satisfies:

$$
\inf _{\lambda \in \sigma(Q(x)) \backslash\left\{\lambda_{2}(x)\right\}}\left|\lambda-\lambda_{2}(x)\right| \geq \delta .
$$

In particular, this last assumption implies that the spectral projector $\pi(x)$ of $Q(x)$ associated to to the wave packet $\left\{\lambda_{1}(x), \lambda_{2}(x)\right\}$ is $C^{2}$-regular with respect to $x$ (see [12]). Furthermore, by the mini-max principle, 
we deduce that $\lambda_{1}(x)$ and $\lambda_{2}(x)$ are uniformly bounded with respect to $x$ and can be analytically extended on $D_{\delta_{0}}$.

We also assume that $\lambda_{2}(x)$ has a potential well above the maximum level of $\lambda_{1}(x)$ :

$$
\lambda_{2}(x) \geq 0, \quad \sup _{x \in \mathbb{R}^{n}} \lambda_{1}(x)<0, \quad \lim _{|x| \rightarrow \mathbb{R}^{n}} \lambda_{2}(x)>0, \lambda_{2}^{-1}(0)=\{0\}, \lambda_{2}^{\prime \prime}(0)>0 .
$$

By virtue of $(H 1), Q\left(e^{\theta} x\right)$ and $P_{\theta}(h)$ can be extended to small enough complex values of $\theta$ as analytic families. We then put a Viriel hypothesis to avoid the resonances coming from the effective potential $\lambda_{1}(x)$ near level 0 :

$$
\sup _{x \in \mathbb{R}^{n}}\left(2 \lambda_{1}(x)+x . \nabla \lambda_{1}(x)\right)<0 .
$$

Let $u_{j}(x, y), j=1,2$, the two eigenfunctions of $Q(x)$ associated to $\lambda_{1}(x)$ and $\lambda_{2}(x)$ respectively, real and normalized in $L^{2}\left(\mathbb{R}_{y}^{p}\right)$. We then consider a Grushin problem that will lead to the Feshbach reduction ( [3]). For $z, \theta \in \mathbb{R}$, let $A_{z}^{\theta}(h)$ the matrix operator defined from $H^{2}\left(\mathbb{R}_{x}^{n} \times \mathbb{R}_{y}^{p}\right) \oplus L^{2}\left(\mathbb{R}_{x}^{n}\right) \oplus L^{2}\left(\mathbb{R}_{x}^{n}\right)$ to $L^{2}\left(\mathbb{R}_{x}^{n} \times \mathbb{R}_{y}^{p}\right) \oplus L^{2}\left(\mathbb{R}_{x}^{n}\right) \oplus L^{2}\left(\mathbb{R}_{x}^{n}\right)$ by:

$$
A_{z}^{\theta}(h)=\left(\begin{array}{ccc}
P_{\theta}(h)-z & u_{1}^{\theta} & u_{2}^{\theta} \\
\left\langle., u_{1}^{\bar{\theta}}\right\rangle_{y} & 0 & 0 \\
\left\langle., u_{2}^{\bar{\theta}}\right\rangle_{y} & 0 & 0
\end{array}\right)
$$

where $u_{j}^{\theta}(x, y)=u_{j}\left(x e^{\theta}, y\right), j=1,2$, and $\langle., .\rangle_{y}$ denotes the inner product in $L^{2}\left(\mathbb{R}_{y}^{p}\right)$. We shall study the extension of $A_{z}^{\theta}(h)$ to $z$ and $\theta$ complexes, for this we have the following results:

$$
Q\left(e^{\theta} x\right)-Q(x)=\left(V\left(x e^{\theta}, y\right)-V(x, y)\right) \in \mathcal{B}\left(L^{2}\left(\mathbb{R}^{n}\right)\right)
$$

and by the assumption $(H 1)$ :

$$
\frac{\partial}{\partial \theta}\left(V\left(x e^{\theta}, y\right)-V(x, y)\right)=e^{\theta} x \cdot \nabla_{x} V\left(x e^{\theta}, y\right)=\mathcal{O}(1)
$$

uniformly with respect to $x, y$ and $\theta$ complex such that $|\theta|$ small enough. Furthermore, for $j=1,2$,

$$
\lambda_{j}\left(x e^{\theta}\right)-\lambda_{j}(x)=\mathcal{O}(|\theta|)
$$

and $u_{j}$ extends into a holomorphic function on $D_{\delta_{0}}$ with values in $H^{2}\left(\mathbb{R}_{y}^{p}\right)$, such that:

$$
\begin{aligned}
\left\|\partial_{x}^{\alpha} u_{j}^{\theta}\right\|_{y} & =\mathcal{O}\left(\left(1+|x|^{2}\right)^{-|\alpha| / 2}\right), \\
\left\|\partial_{x}^{\alpha} u_{j}^{\theta}-\partial_{x}^{\alpha} u_{j}\right\|_{y} & =\mathcal{O}\left(|\theta|\left(1+|x|^{2}\right)^{-|\alpha| / 2}\right)
\end{aligned}
$$

uniformly with respect to $x$ and $\theta$ complex, $|\theta|$ small enough. 
We now define on $L^{2}\left(\mathbb{R}_{x}^{n} \times \mathbb{R}_{y}^{p}\right)$, for $\theta$ complex, $|\theta|$ small enough, the projector $\pi_{\theta}$ by:

$$
\pi_{\theta} u=\left\langle u, u_{1}^{\bar{\theta}}\right\rangle_{y} u_{1}^{\theta}+\left\langle u, u_{2}^{\bar{\theta}}\right\rangle_{y} u_{2}^{\theta}
$$

and $\widehat{\pi_{\theta}}=1-\pi_{\theta}$. It is therefore simple to show, using previous results, that there is a constant $C>0$ such that for $\theta$ complex, $|\theta|$ small enough,

$$
\operatorname{Re}\left\langle\widehat{\pi_{\theta}}\left(P_{\theta}(h)-z\right) \widehat{\pi_{\theta}} u, \widehat{\pi_{\theta}} u\right\rangle_{y} \geq C\left\|\widehat{\pi_{\theta}} u\right\|^{2}
$$

for all $u \in L^{2}\left(\mathbb{R}_{x}^{n} \times \mathbb{R}_{y}^{p}\right)$ and $|z|$ small enough. In particular, the estimate (4.4) shows the existence of a bounded inverse for $\left(P_{\theta}^{\prime}(h)-z\right)$ the restriction of $\widehat{\pi_{\theta}}\left(P_{\theta}(h)-z\right)$ to $\left\{u \in L^{2}\left(\mathbb{R}_{x}^{n} \times \mathbb{R}_{y}^{p}\right): \widehat{\pi_{\theta}} u=u\right\}$. It is then elementary to verify that $A_{z}^{\theta}(h)$ is invertible, and its inverse $A_{z}^{\theta}(h)^{-1}$ is given by ( $\left.[3]\right)$ :

$$
\left(\begin{array}{ccc}
X_{\theta}(z) & u_{1}^{\theta}-X_{\theta}(z) P_{\theta}(h)\left(. u_{1}^{\theta}\right) & u_{2}^{\theta}-X_{\theta}(z) P_{\theta}(h)\left(. u_{2}^{\theta}\right) \\
\left\langle\left(1-P_{\theta}(h) X_{\theta}(z)\right)(.), u_{1}^{\bar{\theta}}\right\rangle_{y} & z-\left\langle Y_{\theta}(z)\left(. u_{1}^{\theta}\right), u_{1}^{\bar{\theta}}\right\rangle_{y} & \left\langle Y_{\theta}(z)\left(. u_{2}^{\theta}\right), u_{1}^{\bar{\theta}}\right\rangle_{y} \\
\left\langle\left(1-P_{\theta}(h) X_{\theta}(z)\right)(.), u_{2}^{\bar{\theta}}\right\rangle_{y} & \left\langle Y_{\theta}(z)\left(. u_{1}^{\theta}\right), u_{2}^{\bar{\theta}}\right\rangle_{y} & z-\left\langle Y_{\theta}(z)\left(. u_{2}^{\theta}\right), u_{2}^{\bar{\theta}}\right\rangle_{y}
\end{array}\right)
$$

where $X_{\theta}(z)=\left(P_{\theta}^{\prime}(h)-z\right)^{-1} \widehat{\pi_{\theta}}$ and $Y_{\theta}(z)=P_{\theta}(h)-P_{\theta}(h) X_{\theta}(z) P_{\theta}(h)$.

$$
F^{\theta}(z)=\left(\begin{array}{cc}
\left\langle Y_{\theta}(z)\left(. u_{1}^{\theta}\right), u_{1}^{\bar{\theta}}\right\rangle_{y} & \left\langle Y_{\theta}(z)\left(. u_{2}^{\theta}\right), u_{1}^{\bar{\theta}}\right\rangle_{y} \\
\left\langle Y_{\theta}(z)\left(. u_{1}^{\theta}\right), u_{2}^{\bar{\theta}}\right\rangle_{y} & \left\langle Y_{\theta}(z)\left(. u_{2}^{\theta}\right), u_{2}^{\bar{\theta}}\right\rangle_{y}
\end{array}\right)
$$

is called the Feshbach operator, it reduces the initial spectral problem to a problem in $L^{2}\left(\mathbb{R}_{x}^{n}\right) \oplus L^{2}\left(\mathbb{R}_{x}^{n}\right)$. It will also serve to show that we have a theory of resonances for $P(h)$. Indeed, we have:

$$
\begin{aligned}
& F^{\theta}(z)=U_{\theta} F U_{\theta}^{-1}+R^{\theta}(z, h) \\
& F=\left(\begin{array}{cc}
-h^{2} \Delta_{x}+\lambda_{1}(x) & 0 \\
0 & -h^{2} \Delta_{x}+\lambda_{2}(x)
\end{array}\right)
\end{aligned}
$$

and

$$
\begin{aligned}
& R^{\theta}(z, h)= \\
& \left(\begin{array}{cc}
-h^{2}\left\langle\Delta_{x} u_{1}^{\theta}, u_{1}^{\bar{\theta}}\right\rangle_{y}-\left\langle Z_{\theta}(z)\left(. u_{1}^{\theta}\right), u_{1}^{\bar{\theta}}\right\rangle_{y} & \left\langle Y_{\theta}(z)\left(. u_{2}^{\theta}\right), u_{1}^{\bar{\theta}}\right\rangle_{y} \\
\left\langle Y_{\theta}(z)\left(. u_{1}^{\theta}\right), u_{2}^{\bar{\theta}}\right\rangle_{y} & -h^{2}\left\langle\Delta_{x} u_{2}^{\theta}, u_{2}^{\bar{\theta}}\right\rangle_{y}-\left\langle Z_{\theta}(z)\left(. u_{2}^{\theta}\right), u_{2}^{\bar{\theta}}\right\rangle_{y}
\end{array}\right)
\end{aligned}
$$

where $Z_{\theta}(z)=P_{\theta}(h)-Y_{\theta}(z)=P_{\theta}(h) X_{\theta}(z) P_{\theta}(h)$, and for all $m \in \mathbb{Z}$

$$
\left\|R^{\theta}(z, h)\right\|_{\mathcal{B}\left(H^{m}\left(\mathbb{R}^{n}\right) \oplus H^{m}\left(\mathbb{R}^{n}\right), H^{m-1}\left(\mathbb{R}^{n}\right) \oplus H^{m-1}\left(\mathbb{R}^{n}\right)\right)}=\mathcal{O}\left(h^{2}\right) .
$$

On the other hand, using $(H 3)$ and $(H 4),\left(\widetilde{F^{\theta}}-z\right)$ is boundedly invertible from $L^{2}\left(\mathbb{R}_{x}^{n}\right) \oplus L^{2}\left(\mathbb{R}_{x}^{n}\right)$ to $H^{2}\left(\mathbb{R}_{x}^{n}\right) \oplus H^{2}\left(\mathbb{R}_{x}^{n}\right)$, for $z$ complex, $|z|$ small enough, where $\widetilde{F^{\theta}}=U_{\theta} F U_{\theta}^{-1}+W, W \in C_{0}^{\infty}\left(\mathbb{R}^{n}\right)$ such that $\operatorname{Re}\left(W+\lambda_{2}^{\theta}(x)\right)>0$. 
Thus, $K^{\theta}(z, h)=\left(R^{\theta}(z, h)-W(x)\right)\left(\widetilde{F^{\theta}}-z\right)^{-1}$ is a compact operator on $L^{2}\left(\mathbb{R}_{x}^{n}\right) \oplus L^{2}\left(\mathbb{R}_{x}^{n}\right)$, for $\theta$ and $z$ complex small enough. $K^{\theta}(z, h)$ depends analytically on $z$ and $\lim _{z \in \mathbb{R}, z \rightarrow-\infty}\left\|K^{\theta}\left(e^{-2 \theta} z, h\right)\right\|=0$. By Theorem 1.2 , we deduce that $\left(I+K^{\theta}(z, h)\right)^{-1}$ is a $z$-meromorphic family for $z$ in a complex neighborhood of 0 . So it is the same for

$$
\left(F^{\theta}(z)-z\right)^{-1}=\left(\widetilde{F^{\theta}}-z\right)\left(I+K^{\theta}(z, h)\right)^{-1}
$$

and (see [3]):

$$
\left(P_{\theta}(h)-z\right)^{-1}=X_{\theta}(z)+A_{+}^{\theta}(z)\left(F^{\theta}(z)-z\right)^{-1} A_{-}^{\theta}(z)
$$

with

$$
\begin{aligned}
& A_{+}^{\theta}(z)=\left(\left(1-X_{\theta}(z) P_{\theta}(h)\right)\left(. u_{1}^{\theta}\right),\left(1-X_{\theta}(z) P_{\theta}(h)\right)\left(. u_{2}^{\theta}\right)\right) \\
& A_{-}^{\theta}(z)=A_{+}^{\theta}(\bar{z})^{*} .
\end{aligned}
$$

We can also deduce by construction that the spectra of $P_{\theta}(h)$ is discrete near 0 and $z \in \Sigma(h)$ if and only if there exists $\theta \in \mathbb{C}, \operatorname{Im} \theta>0,|\theta|$ small enough such that $z \in \sigma_{\text {disc }}\left(F^{\theta}(z)\right)$.

(4.7) shows that $\left(P_{\theta}(h)-z\right)^{-1}$ extends into a meromorphic function in $z$ near 0 , and we have exactly like in $(4.3)$ :

$$
\begin{aligned}
& \bigcup_{\theta \in \mathbb{C},|\theta|<\delta} \bigcup_{\varphi, \psi}\left\{\text { Poles of }\left\langle\left(P_{\theta}(h)-z\right)^{-1} \varphi, \psi\right\rangle_{L^{2}} \bigcap(]-\varepsilon, \varepsilon[+i]-\varepsilon, \varepsilon[)\right\} \\
= & \bigcup_{\theta \in \mathbb{C},|\theta|<\delta}\left\{\sigma\left(P_{\theta}(h)\right) \cap(]-\varepsilon, \varepsilon[+i]-\varepsilon, \varepsilon[)\right\}
\end{aligned}
$$

is the set of resonances $\Sigma(h)$ of the Hamiltonian $P(h)$ in $]-\varepsilon, \varepsilon[+i]-\varepsilon, \varepsilon[, \varepsilon>0$.

Conflicts of Interest: The author(s) declare that there are no conflicts of interest regarding the publication of this paper.

\section{REFERENCES}

[1] Guillopé, L. \& Zworski, M., Polynomial bounds on the number of resonances for some complete spaces of constant negative curvature near infinity, Asymptotic Anal. 11 (1995), 1-22.

[2] Zworski, M., Poisson formulae for resonaces, Séminaire E.D.P., Ecole Polytechnique, Exposé XIII, 1996-1997.

[3] Belmouhoub, S. \& Messirdi, B., Singular Schrödinger Operators Via Grushin Problem Method, Anal. Univ. Oradea Fasc. Matematica, Tom XXIV (1) (2017), 83-91.

[4] Dyatlov, S. \& Zworski, M., Mathematical theory of scattering resonances, Amer. Math. Soc. Providence, Rhode Island, 2019.

[5] Hörmander, L., The Analysis of Linear Partial Differential Operators I. Distribution Theory and Fourier Analysis, Springer Verlag, 1983.

[6] Reed, M. \& Simon, B., Methods of Modern Mathematical Physics, Academic Press, New York, 1978.

[7] Hislop, P.D., Fundamentals of scattering theory and resonances in quantum mechanics, Cubo (Temuco), 14 (03) (2012), 01-39. 
[8] Hellfer, B. \& Martinez, A., Comparaison entre les diverses notions de résonances, Helv. Phys. Acta, 60 (1987), $992-1003$.

[9] Hunziker, W., Distorsion analyticity and molecular resonance curves, Ann. Inst. Henri Poincaré, 45 (1986), 339-358.

[10] Messirdi, B., Asymptotique de Born-Oppenheimer pour la prédissociation moléculaire (cas de potentiels réguliers), Ann. Inst. Henri Poincaré, 61 (1992), 255-292.

[11] Messirdi, B., Asymptotique de Born-Oppenheimer pour la prédissociation moléculaire, Ph. D. Thesis, University of Paris 13, 1993.

[12] Combes, J.M. \& Seiler, R., Regularity and asymptotic properties of the discrete spectrum of electronic hamiltonians, Int. J. Quantum Chem. XIV (1978), 213-229. 